\title{
Characteristics of Dielectric-Line-Loaded Conductor-Backed Slotline (CBS)
}

\author{
Jeng-Wen Huang, Student Member, IEEE, and Ching-Kuang C. Tzuang, Senior Member, IEEE
}

\begin{abstract}
The mode-coupling phenomenon prevents the conventional shielded conductor-backed slotline (CBS) from broadband applications. This paper proposes a modified CBS by adding a dielectric line of higher dielectric constant on the top of the slot region to circumvent the mode-coupling problem. The dispersion characteristics of the resultant dielectric line-loaded CBS are studied by using the full-wave space-domain Green's impedance function approach. The theoretic results show that the particular modified CBS significantly reduces mode-coupling phenomenon and is suitable for broadband applications. Furthermore, it is possible to adjust the values of the propagation constant and the characteristic impedance by changing the thickness of the dielectric line on top of the slot region. Finally, the design curves for the dielectric line-loaded CBS are provided for potential applications.
\end{abstract}

\section{INTRODUCTION}

$\mathbf{T}$ HE CONDUCTOR-BACKED slotline (CBS) circuits have been widely used in uniplanar (M)MIC ((monolithic) microwave integrated circuit) designs [1]-[3]. The CBS, however, suffers from potential problem of leakage due to coupling to the parallel-plate TEM mode and produces unexpected effects [4]. To this end, Das proposed a layered uniform dielectric-loaded CBS configuration to avoid such undesired leakage [5]. A different approach was reported earlier using a suspended slotline of various dielectric substrates [6]. Rigorous investigations showed that leakage phenomena could be reduced in the suspended slotline if the thickness of the lower substrate region of lower dielectric constant was increased [6].

As shown in Fig. 1(a), where $w_{1}$ equals to $w_{2}$, the CBS is symmetric. Otherwise it is asymmetric. When the CBS is symmetric about $x=a / 2$ plane, the fundamental CBS mode and the first higher order parasitic waveguide mode have symmetric planes located at $x=a / 2$ in the form of electric and magnetic walls, respectively. Thus, these two modes will cross in the dispersion curves obtained theoretically and they will not couple to each other. Later, it will be shown that if the CBS is asymmetric, the fundamental mode couples to the parasitic waveguide mode in a mode-coupling manner similar to that reported for the asymmetric CBCPW (conductor-backed coplanar waveguide) [7] (see Fig. 3). This can be categorized as the mode-coupling between two forward (backward) traveling waves as described in [8]. In engineering

Manuscript received May 13, 1994. This work was supported by the National Science Council, Republic of China, under grants NSC 82-0404D009-033 and NSC83-0404-E009-045.

The authors are with the Institute of Communication Engineering National Chiao Tung University, Taiwan, R.O.C.

IEEE Log number 9402972.

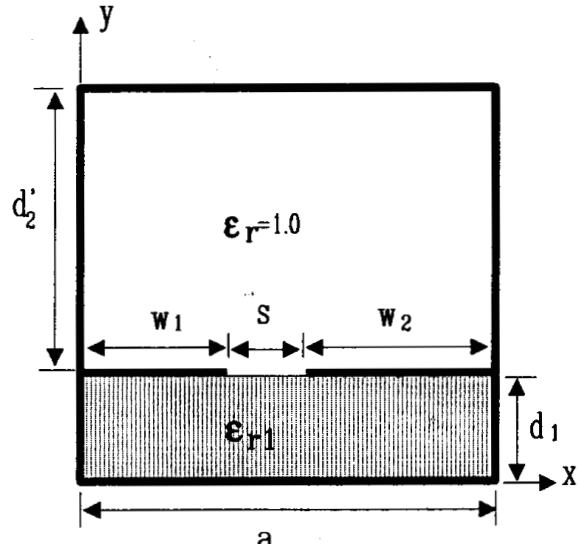

(a)

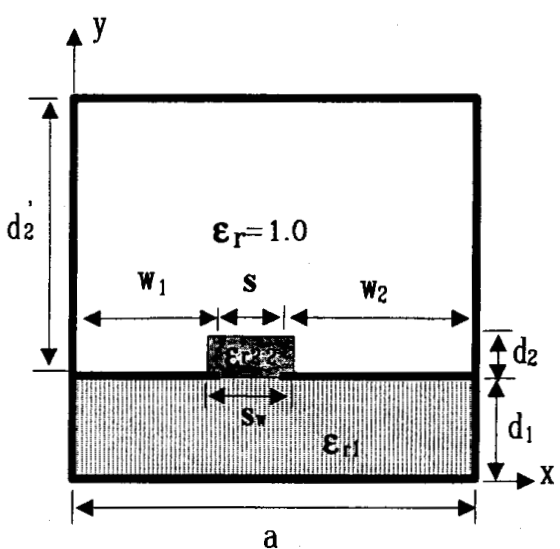

(b)

Fig. 1. Geometry of (a) the conventional shielded CBS and (b) the asymmetric dielectric-line-loaded shielded CBS.

practice, a slight difference in $w_{1}$ and $w_{2}$ will make the CBS asymmetric and the mode-coupling phenomenon is likely to happen, resulting in an asymmetric CBS. Therefore, the aim of this paper is to report a modified CBS guided-structure that will prevent such mode-coupling from happening regardless of the symmetry.

The modified CBS structure that places a finite-width dielectric line of dielectric constant much higher than that of 


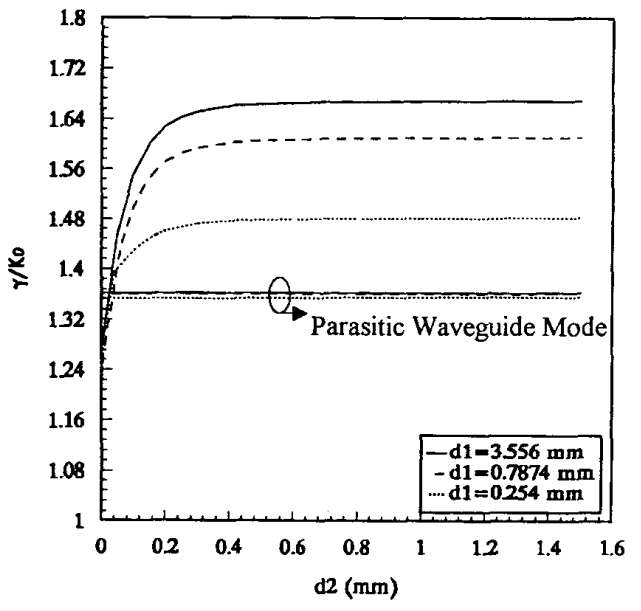

(a)

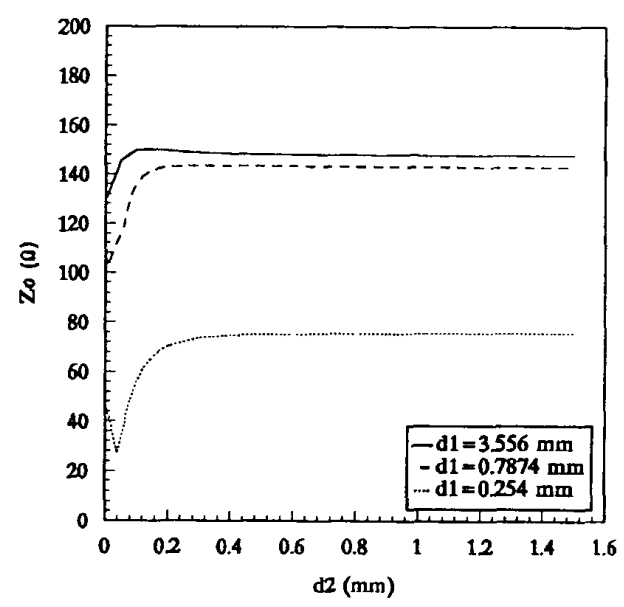

(b)

Fig. 2. (a) Normalized propagation constant and (b) characteristic impedance as a function of dielectric line thickness $d_{2}$ for modified CBS of Fig. 1(b) with three different substrate thickness $(d l=3.556,0.7874$, and $0.254 \mathrm{~mm})$ at $35 \mathrm{GHz} . a=d_{2}^{\prime}=7.112 \mathrm{~mm}, w_{1}=2.8 \mathrm{~mm}, \varepsilon_{r 1}=2.22$, and $s=s_{w}=0.4 \mathrm{~mm}$.

the substrate on top of the slot region of the CBS is proposed in Fig. 1(b). A similar approach had been successfully applied to improve the propagation characteristics of a shielded conductor-backed coplanar waveguide (CBCPW) [7]. Here, we will investigate in detail the propagation characteristics of the proposed modified CBS showing its encouraging guided wave property for broadband application and providing useful information for applying the modified CBS. The theoretic results presented are based on the Green's impedance function approach reported elsewhere [7], [9].

\section{DisPERSion AND GENERAL PROPAGation CHARACTERISTICS OF THE DIELECTRIC-LINE-LOADED CBS}

Fig. 1(b) illustrates the proposed modified CBS. The additional dielectric line of width $s_{w}$ and dielectric constant $\varepsilon_{r 22}$

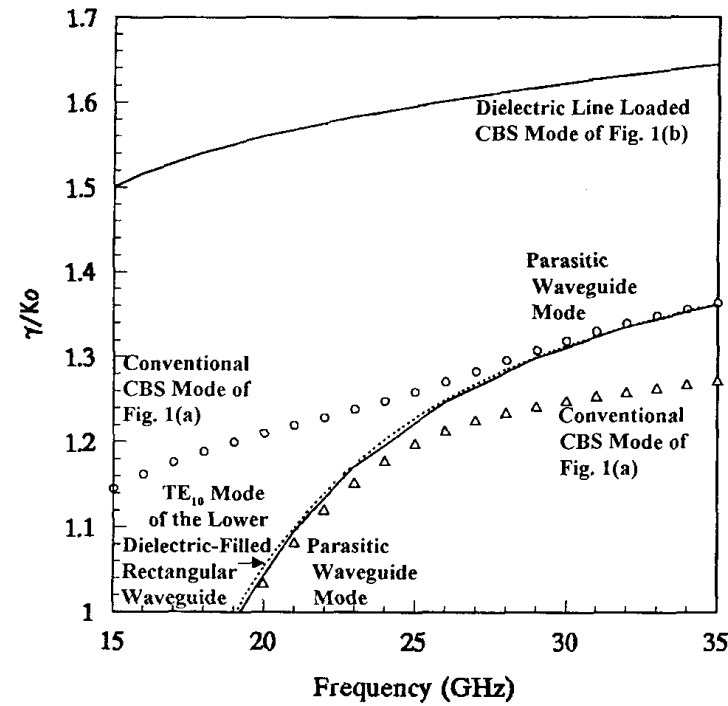

Fig. 3. Illustration of the mode-coupling-free dielectric-line-loaded CBS and the potentially hazardous mode-coupling effect between the conventional CBS mode and the parasitic waveguide mode. $0, \Delta$ : Normalized propagation constant of slotline mode and parasitic waveguide mode of the conventional CBS as shown in Fig. 1(a). ___. Normalized propagation constant of slotline mode and parasitic waveguide mode of the dielectric-line-loaded CBS as shown in Fig. 1(b). . . . . TE 10 mode of the lower dielectric-filled rectangular waveguide assuming that $s=0$. The structural parameters are $a=d_{2}^{\prime}=7.112 \mathrm{~mm}, d_{1}=3.556 \mathrm{~mm}, d_{2}=0.254 \mathrm{~mm}, w_{1}=2.8 \mathrm{~mm}, \varepsilon_{r 1}=2.22$, $\varepsilon_{r 22}=10.2$, and $s=s_{w}=0.4 \mathrm{~mm}$.

alters the propagation characteristics significantly. The exact width $s_{w}$ is not too critical. This paper assumes that the gap of slotline is equal to $s_{w}\left(s=s_{w}\right)$. The general propagation characteristics of Fig. 1(b) can be understood in at least two ways. One is at a fixed operating frequency while changing the structural dimensions; the other is the dispersion characteristics of the modified CBS using certain structural dimensions. The former is shown in Fig. 2(a) and (b), which plots the normalized propagation constant and characteristic impedance (power-voltage definition), respectively, with the structural and material constants given in the same plot. Fig. 2(a) shows the variation of the normalized propagation constant versus the thickness $d_{2}$ of the dielectric line for three different substrate thickness from relatively thin $\left(d_{1}=0.254 \mathrm{~mm}, 10\right.$ mils $)$ to very thick $\left(d_{1}=3.556 \mathrm{~mm}, 140\right.$ mils $)$ dielectric layers at $35 \mathrm{GHz}$. The corresponding variation of the characteristic impedance is shown in Fig. 2(b). By increasing the thickness of the dielectric line $\left(d_{2}\right)$, one clearly sees a rapid rise and subsequent flatness of the normalized propagation constant and the characteristic impedance for all three different substrate thickness. The normalized propagation constant of the first waveguide mode, however, is negligibly affected by the additional dielectric line. Therefore, it is likely that by adding a thin dielectric line, typically $d_{2}$ greater than $0.2 \mathrm{~mm}$, one can effectively prevent mode coupling of the fundamental slotline mode and the parasitic waveguide mode.

With $\varepsilon_{r 22}=10.2$, Fig. 3 manifests the fact that the particular dielectric-line-loaded CBS exhibits very smooth and unper- 


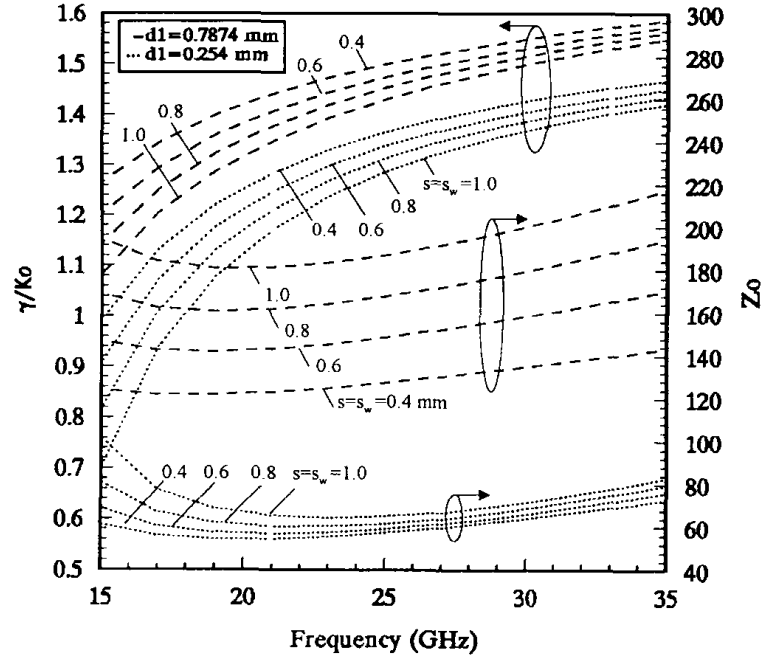

Fig. 4. Dispersion characteristics of the dielectric-line-loaded CBS of Fig l(b) with respect to slot width $S=S_{w}$ and two different substrate thickness ( $d_{1}=0.254$ and $0.7874 \mathrm{~mm}$ ). $a=d_{2}^{\prime}=7.112 \mathrm{~mm}, d_{2}=0.254 \mathrm{~mm}, w_{1}=2.8 \mathrm{~mm}$, $\Xi_{r 1}=2.22$, and $\xi_{r 22}=10.2$.

turbed dispersion characteristics from which no mode-coupling may exist in the frequency band of interest. On the contrary, without adding the dielectric line, the conventional CBS mode couples to the parasitic waveguide mode, resembling $T E_{10}$ mode, at the neighborhood of $26 \mathrm{GHz}$. The modecoupling between these two modes jeopardizes the broadband application of the conventional CBS mode. Figs. 2 and 3 also imply that the cut-off frequency of the modified CBS is lower than that of the conventional CBS. The modified CBS is therefore suitable for broadband application.

\section{Design Curves for the Dielectric-Line-LoAded CBS}

Fig. 4 plots the dispersion characteristics of the dielectricline-loaded CBS using 10 and 31 mils substrates. The rapid change of the propagation characteristics with respect to the change of $d_{2}$ as illustrated in Fig. 2 suggests that the thickness of $d_{2}$ can be 10 mils $(0.254 \mathrm{~mm})$. In other words, for those guided structures with $d_{2}$ greater than 10 mils, the propagation characteristics remain relatively unchanged. Using the gap with $S$ and consequently $s_{w}$ as the controlling parameters, one may select the desired gap width for particular design.

\section{CONCLUSION}

Rigorous theoretic investigation of the proposed dielectricline-loaded conductor-backed slotline (CBS) is presented. The results show that the modified CBS is suitable for broadband circuit design in a sense that the mode-coupling phenomenon that would occur in the conventional CBS can be suppressed. Useful design informations and general propagation characteristics of the dielectric-line-loaded CBS are also provided for potential applications. Not shown in Fig. 3, however, is the mode coupling that will take place between the slotline mode and the dielectric waveguide mode at very high frequency, i.e., $74.6 \mathrm{GHz}$ in our particular case study with the structural parameters and material constants given in Fig. 3.

\section{REFERENCES}

[1] H. Ogawa and A. Minagawa, "Uniplanar MIC balanced multiplier-a proposed new structure for MIC's," IEEE Trans. Microwave Theory Tech., vol. 35, pp. 1363-1368, Dec. 1987.

[2] T. Hirota, Y. Tarusawa, and H. Ogawa, "Uniplanar MMIC hybrids-a proposed new MMIC structure," IEEE Trans. Microwave Theory Tech. vol. 35, pp. 576-581, June 1987.

[3] D. Cahana, "A new coplanar waveguide/slotline double-balanced mixer," IEEE MTT-S Dig., pp. 967-968, 1989.

[4] H. Sigesawa, M. Tsuji, and A. A. Oliner, "Conductor backed slotline and coplanar waveguide: dangers and fullwave analyses," IEEE MTT-S Dig., pp. 199-202, 1988.

[5] N. K. Das and D. M. Pozar, "Full-wive spectral-domain computation of material, radiation, and guided wave losses in infinite multilayered printed transmission lines," IEEE Trans. Microwave Theory Tech., vol. 39 , pp. $54-63,1991$.

[6] R. W. Jackson, "Mode conversion at discontinuities in finite width conductor backed coplanar waveguide," IEEE Trans. Microwave Theory Tech., vol. 37, pp. 1582-1589, Oct. 1989.

[7] J.-W. Huang and C.-K. C. Tzuang, "Mode-coupling-avoidance of shielded conductor-backed coplanar waveguide (CBCPW) using dielectric lines compensation," in 1994 Int. Microwave Symp. Dig. pp. 149-152.

[8] C.-K. C Tzuang and J.-M. Lin, "On the mode-coupling formation of complex modes," IEEE Trans. Microwave Theory Tech., vol 41, pp. 1400-1408, Aug. 1993.

[9] J.-W. Huang and C.-K. C. Tzuang, "Green's impedance function approach for propagation characteristics of generalized striplines and slotlines on non-layered substrates," to appear in IEEE Trans. Microwave Theory Tech., Dec. 1994. 